\title{
Fourier Transforms of Finite Chirps
}

\author{
Peter G. Casazza ${ }^{1}$ and Matthew Fickus ${ }^{2}$ \\ ${ }^{1}$ Department of Mathematics, University of Missouri, Columbia, MO 65211, USA \\ ${ }^{2}$ Department of Mathematics and Statistics, Air Force Institute of Technology, Wright-Patterson AFB, OH 45433, USA
}

Received 15 October 2004; Revised 27 February 2005; Accepted 5 April 2005

\begin{abstract}
Chirps arise in many signal processing applications. While chirps have been extensively studied as functions over both the real line and the integers, less attention has been paid to the study of chirps over finite groups. We study the existence and properties of chirps over finite cyclic groups of integers. In particular, we introduce a new definition of a finite chirp which is slightly more general than those that have been previously used. We explicitly compute the discrete Fourier transforms of these chirps, yielding results that are number-theoretic in nature. As a consequence of these results, we determine the degree to which the elements of certain finite tight frames are well distributed.
\end{abstract}

Copyright @ 2006 Hindawi Publishing Corporation. All rights reserved.

\section{INTRODUCTION}

A linear chirp is a function whose frequency changes linearly with time. For example, while a wave function of the form $\exp (2 \pi \mathrm{i} x t)$ has constant frequency $x$, the chirp $\exp (2 \pi \mathrm{i}(x t+$ $\left.y t^{2} / 2\right)$ ) has frequency $x+y t$ at time $t \in \mathbb{R}$. Chirps often arise in nature as a consequence of the Doppler effect, the phenomenon by which the perceived frequency of a wave is altered whenever the wave is emanating from or reflecting off a moving body. As such, chirps have historically been of great interest in applications such as radar and sonar.

However, the study of chirps has mostly been confined to the real line and the integers, in the context of integral transforms and the chirp Z -transform, respectively. Less attention has been paid to the study of chirps over finite cyclic groups, that is, to chirps over $\mathbb{Z}_{a} \equiv \mathbb{Z} / a \mathbb{Z}=\{0, \ldots, a-1\}$, where $a$ is a positive integer. This is in contrast to wave functions which, in the context of Fourier transforms, have been studied for many decades on arbitrary locally compact abelian groups.

At the same time, the concept of a finite chirp is by no means new. An excellent discussion of a modern application of finite chirps is given in [1], in which a discrete chirp-Fourier transform is introduced. Chirplets have been used in image processing for over a decade [2]. For that matter, discrete chirps, under a different name, were investigated by Gauss in his study of quadratic reciprocity. Indeed, the computation of Gauss sums [3] is equivalent to finding the discrete Fourier transform (DFT) of a finite chirp, and was a subject of great interest in the mid-nineteenth century. This connection between modern signal processing and classical number theory is emphasized in [4], in which the trace of the DFT matrix,

$$
\operatorname{Tr}\left(\mathrm{F}_{a}\right)=\frac{1}{\sqrt{a}} \sum_{t=0}^{a-1} \mathrm{e}^{2 \pi \mathrm{i} t^{2} / a}
$$

is noted to be the canonical example of a Gauss sum. An independent derivation of this trace is given in [5], giving an interesting example of when the concepts of applied signal processing may be used to prove results in pure mathematics.

Section 2 provides a brief introduction to the mathematics of finite signal processing, with an emphasis upon the finite form of the Poisson summation formula. In Section 3, we introduce our definition of finite chirps and discuss their elementary properties. In Section 4, we begin the task of computing the Fourier transforms of our chirps. In particular, we compute the magnitudes of the Fourier coefficients and discuss their significance with respect to finite tight frames. Finally, in Section 5, we complete our derivation of the Fourier transforms of these chirps.

\section{PRELIMINARIES}

Let $\mathbb{Z}^{+}$be the set of all positive integers. For any $a \in \mathbb{Z}^{+}$, let $\mathbb{Z}_{a} \equiv \mathbb{Z} / a \mathbb{Z}$ be the finite group of integers in which addition is performed modulo $a$. Consider the space of all complexvalued functions over $\mathbb{Z}_{a}$,

$$
\ell\left(\mathbb{Z}_{a}\right)=\left\{f: \mathbb{Z}_{a} \longrightarrow \mathbb{C}\right\},
$$

in which function addition, scalar multiplication, and the inner product are defined in the usual fashion. We will 
equivalently regard elements of $\ell\left(\mathbb{Z}_{a}\right)$ as $a$-periodic, complexvalued sequences over $\mathbb{Z}$ so as to consider $f(t)$ for any $t \in$ $\mathbb{Z}$. The Dirac basis for $\ell\left(\mathbb{Z}_{a}\right)$ consists of the delta functions $\left\{\delta_{a}^{x}\right\}_{x \in \mathbb{Z}_{a}}$,

$$
\delta_{a}^{x}(t)=\left\{\begin{array}{ll}
1, & t=x \\
0, & t \neq x
\end{array} \quad \bmod a,\right.
$$

and is an orthonormal basis for $\ell\left(\mathbb{Z}_{a}\right)$. Meanwhile, the Fourier basis for $\ell\left(\mathbb{Z}_{a}\right)$ consists of the wave functions $\left\{\mathrm{m}_{a}^{x}\right\}_{x \in \mathbb{Z}_{a}}$,

$$
\mathrm{m}_{a}^{x}(t)=\mathrm{e}^{2 \pi \mathrm{i} x t / a}=\mathrm{w}_{a}^{x t},
$$

where $\mathrm{w}_{a} \equiv \mathrm{e}^{2 \pi \mathrm{i} / a}$ is the "first" ath root of unity. When suitably scaled by a factor of $\sqrt{a}$, the Fourier basis is also an orthonormal basis for $\ell\left(\mathbb{Z}_{a}\right)$.

For $x \in \mathbb{Z}$, the corresponding translation and modulation operators are $\mathrm{T}_{a}^{x}, \mathrm{M}_{a}^{x}: \ell\left(\mathbb{Z}_{a}\right) \rightarrow \ell\left(\mathbb{Z}_{a}\right)$, respectively, where,

$$
\begin{aligned}
\left(\mathrm{T}_{a}^{x} f\right)(t) & =f(t-x), \\
\left(\mathrm{M}_{a}^{x} f\right)(t) & =\mathrm{m}_{a}^{x}(t) f(t) .
\end{aligned}
$$

Next, the Fourier transform on $\mathbb{Z}_{a}$ is $\mathrm{F}_{a}: \ell\left(\mathbb{Z}_{a}\right) \rightarrow \ell\left(\mathbb{Z}_{a}\right)$,

$$
\left(\mathrm{F}_{a} f\right)(x)=\frac{1}{\sqrt{a}} \sum_{t \in \mathbb{Z}_{a}} f(t) \mathrm{w}_{a}^{-x t}
$$

Finally, the product and convolution of any two elements $f, g \in \ell\left(\mathbb{Z}_{a}\right)$ are $f g, f * g \in \ell\left(\mathbb{Z}_{a}\right)$, respectively, where

$$
\begin{aligned}
(f g)(t) & =f(t) g(t), \\
(f * g)(t) & =\sum_{s \in \mathbb{Z}_{a}} f(t-s) g(s) .
\end{aligned}
$$

Several well-known properties of these operations are summarized in the following result. Due to their ubiquity, these properties are stated without proof, and will be used throughout our work without explicit reference.

Proposition 1. For any $f, g \in \ell\left(\mathbb{Z}_{a}\right)$ and any $x, y \in \mathbb{Z}$,

(1) $\mathrm{M}_{a}^{x} \mathrm{~T}_{a}^{y}=\mathrm{m}_{a}^{y}(x) \mathrm{T}_{a}^{y} \mathrm{M}_{a}^{x}$,

(2) $\mathrm{F}_{a} \mathrm{~T}_{a}^{x}=\mathrm{M}_{a}^{-x} \mathrm{~F}_{a}$,

(3) $\mathrm{F}_{a} \mathrm{M}_{a}^{x}=\mathrm{T}_{a}^{x} \mathrm{~F}_{a}$,

(4) $\mathrm{F}_{a}(f g)=(1 / \sqrt{a})\left(\mathrm{F}_{a} f\right) *\left(\mathrm{~F}_{a} g\right)$,

(5) $\mathrm{F}_{a}(f * g)=\sqrt{a}\left(\mathrm{~F}_{a} f\right)\left(\mathrm{F}_{a} g\right)$.

We will also make use of a finite version of the Poisson summation formula. Though not nearly as well known as its infinite-dimensional cousins, this version is easily proved using finite geometric series. Here and throughout, we write $x \mid y$ to denote integers $x$ and $y$ such that $x$ divides $y$.

Proposition 2. Let $a, b \in \mathbb{Z}^{+}$. Then, for all $f \in \ell\left(\mathbb{Z}_{a b}\right)$,

$$
\underset{\left\{t \in \mathbb{Z}_{a b}, a \mid t\right\}}{\sqrt{a} \sum_{\left\{x \in \mathbb{Z}_{a b}, b \mid x\right\}} f(t)}=\sqrt{b} \sum_{a b}\left(\mathrm{~F}_{a b} f\right)(x) .
$$

\section{FINITE CHIRPS}

Perhaps the most difficult aspect of finite chirps is finding their "proper" definition. In [1], the lowest-order nontrivial finite chirp is defined to be $f \in \ell\left(\mathbb{Z}_{a}\right)$,

$$
f(t)=\mathrm{w}_{a}^{t^{2}}=\mathrm{e}^{2 \pi \mathrm{i} t^{2} / a} .
$$

We note that this chirp is well defined over $\mathbb{Z}_{a}$, that is,

$$
\begin{aligned}
f(t+a) & =\mathrm{e}^{2 \pi \mathrm{i}(t+a)^{2} / a} \\
& =\mathrm{e}^{2 \pi t^{2} / a} \mathrm{e}^{2 \pi \mathrm{i}(2 t+a)} \\
& =\mathrm{e}^{2 \pi \mathrm{i}^{2} / a} 1 \\
& =f(t) .
\end{aligned}
$$

However, in the case of continuous chirps, we note that the squared term in the exponent is usually accompanied by an additional factor of $1 / 2$. This is done to compensate for the fact that the frequency of a wave is obtained by looking at the derivative of the wave function. From this perspective, the finite chirp should instead be defined as

$$
g(t)=\mathrm{w}_{a}^{t^{2} / 2}=\mathrm{e}^{\pi \mathrm{i} t^{2} / a} .
$$

Indeed, much of the original work on Gauss sums [3] takes $g(t)$ as the "canonical" chirp rather than $f(t)$. However, under this definition,

$$
\begin{aligned}
g(t+a) & =\mathrm{e}^{\pi \mathrm{i}(t+a)^{2} / a} \\
& =\mathrm{e}^{\pi \mathrm{i} t^{2} / a} \mathrm{e}^{\pi \mathrm{i}(2 t+a)} \\
& =\mathrm{e}^{\pi \mathrm{i} t^{2} / a}(-1)^{a} \\
& =(-1)^{a} g(t),
\end{aligned}
$$

and thus $g$ is not well defined over $\mathbb{Z}_{a}$ when $a$ is odd. When dealing with Gauss sums, this problem is usually avoided by simply summing over $\{0, \ldots, a-1\}$ rather than an arbitrary collection of coset representatives. This approach is inadequate from the point of view of harmonic analysis and signal processing as one often needs to make translation-based changes of variables in various sums.

We remedy the problem with $g(t)$ in another way: by replacing the quadratic $t^{2}$ with $t(t-a)$. That is, we consider $c_{a} \in \ell\left(\mathbb{Z}_{a}\right)$,

$$
\mathrm{c}_{a}(t)=\mathrm{w}_{a}^{t(t-a) / 2}=\mathrm{e}^{\pi \mathrm{i} t(t-a) / a}
$$

This definition was originally proposed by Kaiblinger [6], as was presented publicly at the conference "Harmonic Analysis and Applications" in honor of John J. Benedetto's 60th birthday. As Kaiblinger noted, this chirp is well defined since

$$
\begin{aligned}
\mathrm{c}_{a}(t+a) & =\mathrm{e}^{\pi \mathrm{i}(t+a) t / a} \\
& =\mathrm{e}^{\pi \mathrm{i} t^{2} / a}(-1)^{t} \\
& =\mathrm{e}^{\pi \mathrm{i} t^{2} / a}(-1)^{-t} \\
& =\mathrm{e}^{\pi \mathrm{i} t(t-a) / a} \\
& =\mathrm{c}_{a}(t) .
\end{aligned}
$$


A similar technique may be used to produce discrete versions of $\mathrm{e}^{2 \pi \mathrm{i}^{n} / n}$ for any $n \in \mathbb{Z}^{+}$. For example, when $n=3$ the function $\mathrm{e}^{2 \pi \mathrm{i} t(t-a)(t-2 a) / 3 a}$ is well defined over $\mathbb{Z}_{a}$.

Returning to the quadratic, we have that for any $x \in \mathbb{Z}$, we may raise $\mathrm{c}_{a}$ to the $x$ th power in a pointwise fashion in order to obtain a well defined $x$ th-order chirp on $\mathbb{Z}_{a}$,

$$
\mathrm{c}_{a}^{x}(t)=\mathrm{w}_{a}^{x t(t-a) / 2}=(-1)^{x t} \mathrm{w}_{a}^{x t^{2} / 2},
$$

and with it, a chirp-modulation operator $\mathrm{C}_{a}^{x}: \ell\left(\mathbb{Z}_{a}\right) \rightarrow \ell\left(\mathbb{Z}_{a}\right)$,

$$
\left(\mathrm{C}_{a}^{x} f\right)(t)=\mathrm{c}_{a}^{x}(t) f(t) .
$$

We note that for even integers $x, \mathrm{c}_{a}^{x}$ reduces to a power of the finite chirp given in (9), and thus our definition is in fact an extension of that given in [1]. We now state and prove some elementary properties of these chirps.

Proposition 3. For $a \in \mathbb{Z}^{+}$and any $t, x, y \in \mathbb{Z}$,

(1) $\overline{\mathrm{c}_{a}^{x}}=\mathrm{c}_{a}^{-x}$,

(2) $\mathrm{c}_{a}^{x+a}=\mathrm{c}_{a}^{x}$ when $a$ is odd,

(3) $\mathrm{c}_{a}^{x+a}=\mathrm{m}_{a}^{a / 2} \mathrm{c}_{a}^{x}$ when $a$ is even,

(4) $\mathrm{c}_{a}^{x}(y t)=\mathrm{c}_{a}^{x y^{2}}(t)$,

(5) $\mathrm{c}_{a}^{x}(-t)=\mathrm{c}_{a}^{x}(t)$,

(6) $\mathrm{c}_{a}^{y}(t-x)=\mathrm{c}_{a}^{y}(t) \mathrm{m}_{a}^{-x y}(t) \mathrm{c}_{a}^{y}(x)$,

(7) $\mathrm{T}_{a}^{x} \mathrm{C}_{a}^{y}=\mathrm{c}_{a}^{y}(x) \mathrm{M}_{a}^{-x y} \mathrm{C}_{a}^{y} \mathrm{~T}_{a}^{x}$,

(8) $\mathrm{M}_{a}^{x} \mathrm{C}_{a}^{y}=\mathrm{C}_{a}^{y} \mathrm{M}_{a}^{x}$.

Proof. (1) This follows immediately from the fact that $\overline{\mathrm{w}_{a}}=$ $\mathrm{w}_{a}^{-1}$.

(2)-(3) Since $t \in \mathbb{Z}$ is even if and only if $t^{2}$ is even,

$$
\mathrm{w}_{a}^{a t^{2} / 2}=\mathrm{e}^{\pi \mathrm{i} a t^{2} / a}=(-1)^{t^{2}}=(-1)^{t} .
$$

Therefore, for any $a \in \mathbb{Z}$, we have

$$
\begin{aligned}
\mathrm{c}_{a}^{x+a}(t) & =(-1)^{(x+a) t} \mathrm{w}_{a}^{(x+a) t^{2} / 2} \\
& =(-1)^{x t} \mathrm{w}_{a}^{x t^{2} / 2}(-1)^{a t} \mathrm{w}_{a}^{a t^{2} / 2} \\
& =\mathrm{c}_{a}^{x}(t)(-1)^{a t}(-1)^{t} \\
& =(-1)^{(a+1) t} \mathrm{c}_{a}^{x}(t) .
\end{aligned}
$$

Thus, when $a$ is odd, $\mathrm{c}_{a}^{x+a}(t)=\mathrm{c}_{a}^{x}(t)$. If, on the other hand, $a$ is even, then $a / 2 \in \mathbb{Z}$ and

$$
\mathrm{c}_{a}^{x+a}(t)=(-1)^{t} \mathrm{c}_{a}^{x}(t)=\mathrm{e}^{2 \pi \mathrm{i}(a / 2) t / a} \mathrm{c}_{a}^{x}(t)=\mathrm{m}_{a}^{a / 2} \mathrm{c}_{a}^{x} .
$$

(4) As before, note that $(-1)^{y}=(-1)^{y^{2}}$. Thus,

$$
\mathrm{c}_{a}^{x}(y t)=(-1)^{x y t} \mathrm{w}_{a}^{x(y t)^{2} / 2}=(-1)^{x y^{2} t} \mathrm{w}_{a}^{x y^{2} t^{2} / 2}=\mathrm{c}_{a}^{x y^{2}}(t) .
$$

(5) Apply the previous result to $y=-1$.

(6) Simply note that

$$
\begin{aligned}
\mathrm{c}_{a}^{y}(t-x) & =(-1)^{y(t+x)} \mathrm{w}_{a}^{y(t-x)^{2} / 2} \\
& =(-1)^{y t}(-1)^{y x} \mathrm{w}_{a}^{y t^{2} / 2} \mathrm{w}_{a}^{-y x t} \mathrm{w}_{a}^{y x^{2} / 2} \\
& =\mathrm{c}_{a}^{y}(t) \mathrm{m}_{a}^{-x y}(t) \mathrm{c}_{a}^{y}(x) .
\end{aligned}
$$

(7) This follows immediately from the previous result.

(8) This follows immediately from the fact that both $\mathrm{M}_{a}^{x}$ and $\mathrm{C}_{a}^{y}$ are multiplicative operators.

We next discuss a simple way in which our finite chirps may be used to provide overcomplete decompositions of elements of $\ell\left(\mathbb{Z}_{a}\right)$. In particular, we consider the collection of $a^{2}$ functions obtained by taking every modulation of $\left\{c_{a}^{y}\right\}_{y=0}^{a-1}$. That is, we consider the collection

$$
\left\{\mathrm{m}_{a}^{x} \mathrm{c}_{a}^{y}\right\}_{y=0, x \in \mathbb{Z}_{a}}^{a-1}
$$

Note that by the previous result, this collection is "complete," namely, it contains every possible modulation of every possible chirp. Furthermore, this collection is effectively translation invariant, as the translation of any element is a unitscalar multiple of another element.

The next result shows that this collection is a finite tight frame for $\ell\left(\mathbb{Z}_{a}\right)$, and thus possesses an exceptionally efficient means by which any $f \in \ell\left(\mathbb{Z}_{a}\right)$ may be decomposed into a linear combination of the collection's elements. The argument used is essentially the same idea used to show that any union of orthonormal bases is a tight frame.

Proposition 4. For any $a \in \mathbb{Z}^{+}$,

$$
f=\frac{1}{a^{2}} \sum_{y=0}^{a-1} \sum_{x \in \mathbb{Z}_{a}}\left\langle f, \mathrm{~m}_{a}^{x} \mathrm{c}_{a}^{y}\right\rangle \mathrm{m}_{a}^{x} \mathrm{c}_{a}^{y}
$$

for all $f \in \ell\left(\mathbb{Z}_{a}\right)$.

Proof. We begin by recalling that the normalized Fourier basis $\left\{(1 / \sqrt{a}) \mathrm{m}_{a}^{x}\right\}_{x \in \mathbb{Z}_{a}}$ is an orthonormal basis for $\ell\left(\mathbb{Z}_{a}\right)$. For any $y \in \mathbb{Z}$, the fact that $\left|c_{a}^{y}(t)\right|=1$ for all $t \in \mathbb{Z}_{a}$ automatically implies that $\left\{(1 / \sqrt{a}) \mathrm{m}_{a}^{x} \mathrm{c}_{a}^{y}\right\}_{x \in \mathbb{Z}_{a}}$ is also an orthonormal basis for $\ell\left(\mathbb{Z}_{a}\right)$. Thus, for any $y \in \mathbb{Z}$, Parseval's identity gives

$$
a f=\sum_{x \in \mathbb{Z}_{a}}\left\langle f \mathrm{~m}_{a}^{x} c_{a}^{y}\right\rangle \mathrm{m}_{a}^{x} \mathrm{c}_{a}^{y}
$$

for all $f \in \ell\left(\mathbb{Z}_{a}\right)$. Summing these orthonormal basis decompositions over all $y=0, \ldots, a-1$ gives

$$
a^{2} f=\sum_{y=0}^{a-1} \sum_{x \in \mathbb{Z}_{a}}\left\langle f, \mathrm{~m}_{a}^{x} \mathrm{c}_{a}^{y}\right\rangle \mathrm{m}_{a}^{x} \mathrm{c}_{a}^{y}
$$

for all $f \in \ell\left(\mathbb{Z}_{a}\right)$.

We conclude this section by noting that not all tight frames are equally useful. As discussed in detail in [7], tight frames tend to perform better in applications when the frame elements are designed to be as uncorrelated as possible. In our context, the degree to which our frame elements are uncorrelated is measured by the quantity

$$
\max _{\left(x_{1}, y_{1}\right) \neq\left(x_{2}, y_{2}\right)}\left|\left\langle\mathrm{m}_{a}^{x_{1}} \mathrm{c}_{a}^{y_{1}}, \mathrm{~m}_{a}^{x_{2}} \mathrm{c}_{a}^{y_{2}}\right\rangle\right|
$$

As demonstrated below, this quantity may be explicitly computed by first finding the magnitudes of the discrete Fourier transforms of an arbitrary finite chirp. 


\section{INNER PRODUCTS OF MODULATED CHIRPS}

The remainder of this paper is dedicated to the computation of the Fourier transforms of our finite chirps. The following result, though simple, demonstrates the advantage of having an extra " $1 / 2$ " term present in the definition of our chirps, as it allows us to easily complete a square that arises in the exponents.

Lemma 1. For any $a \in \mathbb{Z}^{+}$and any $b, x, y \in \mathbb{Z}$,

$$
\left(\mathrm{F}_{a} \mathrm{c}_{a}^{b}\right)(x+b y)=\overline{\mathrm{m}_{a}^{x}(y) \mathrm{c}_{a}^{b}(y)}\left(\mathrm{F}_{a} \mathrm{c}_{a}^{b}\right)(x) .
$$

Proof. We first note that

$$
\begin{aligned}
\left(\mathrm{F}_{a} \mathrm{c}_{a}^{b}\right)(x+b y) & =\frac{1}{\sqrt{a}} \sum_{t \in \mathbb{Z}_{a}} \mathrm{c}_{a}^{b}(t) \mathrm{w}_{a}^{-(x+b y) t} \\
& =\frac{1}{\sqrt{a}} \sum_{t \in \mathbb{Z}_{a}}(-1)^{b t} \mathrm{w}_{a}^{b t^{2} / 2} \mathrm{w}_{a}^{-x t} \mathrm{w}_{a}^{-b y t} .
\end{aligned}
$$

Next, we complete the square in the exponents, that is, we observe that $b t^{2}-2 b y t=b\left[(t-y)^{2}-y^{2}\right]$, and therefore

$$
\begin{aligned}
\left(\mathrm{F}_{a} \mathrm{c}_{a}^{b}\right) & (x+b y) \\
= & \frac{1}{\sqrt{a}} \sum_{t \in \mathbb{Z}_{a}}(-1)^{b t} \mathrm{w}_{a}^{b\left[(t-y)^{2}-y^{2}\right] / 2} \mathrm{w}_{a}^{-x t} \\
= & (-1)^{b y} \mathrm{w}_{a}^{-b y^{2} / 2} \frac{1}{\sqrt{a}} \sum_{t \in \mathbb{Z}_{a}}(-1)^{b(t-y)} \mathrm{w}_{a}^{b(t-y)^{2} / 2} \mathrm{w}_{a}^{-x t} \\
= & \overline{\mathrm{c}_{a}^{b}(y)} \frac{1}{\sqrt{a}} \sum_{t \in \mathbb{Z}_{a}}(-1)^{b t} \mathrm{w}_{a}^{b t^{2} / 2} \mathrm{w}_{a}^{-x(t+y)} \\
= & \overline{\mathrm{c}_{a}^{b}(y)} \frac{1}{\sqrt{a}} \sum_{t \in \mathbb{Z}_{a}} \mathrm{c}_{a}^{b}(t) \mathrm{w}_{a}^{-x(t+y)} .
\end{aligned}
$$

To conclude, note that since $c_{a}^{b}$ is well defined over $\mathbb{Z}_{a}$, we may legitimately make a change of variables by translation:

$$
\begin{aligned}
\left(\mathrm{F}_{a} \mathrm{c}_{a}^{b}\right)(x+b y) & =\mathrm{w}_{a}^{-y x} \overline{\mathrm{c}_{a}^{b}(y)} \frac{1}{\sqrt{a}} \sum_{t \in \mathbb{Z}_{a}} \mathrm{c}_{a}^{b}(t) \mathrm{w}_{a}^{-x t} \\
& =\overline{\mathrm{m}_{a}^{x}(y) \mathrm{c}_{a}^{b}(y)}\left(\mathrm{F}_{a} \mathrm{c}_{a}^{b}\right)(x) .
\end{aligned}
$$

The next result concerns the magnitudes of the Fourier transforms of our chirps. Here and throughout, we write $(a, b)$ to denote the greatest common divisor of $a, b \in \mathbb{Z}$. This result also requires us to make the following definition: two integers $a$ and $b$ have a common power of two if there exist odd integers $\alpha$ and $\beta$ such that $a=2^{m} \alpha$ and $b=2^{m} \beta$ for some positive integer $m$. That is, $a$ and $b$ have a common power of two if the exponents of two in their respective prime factorizations are equal.

Proposition 5. Let $a \in \mathbb{Z}^{+}, b \in \mathbb{Z}$ have the greatest common divisor $(a, b)$. If $a$ and $b$ do not have a common power of two,

$$
\left|\left(\mathrm{F}_{a} \mathrm{c}_{a}^{b}\right)(x)\right|^{2}= \begin{cases}(a, b), & x \in(a, b) \mathbb{Z}, \\ 0, & x \notin(a, b) \mathbb{Z} .\end{cases}
$$

Alternatively, if $a$ and $b$ do have a common power of two,

$$
\left|\left(\mathrm{F}_{a} \mathrm{c}_{a}^{b}\right)(x)\right|^{2}= \begin{cases}(a, b), & x \in(a, b)\left(\mathbb{Z}+\frac{1}{2}\right), \\ 0, & x \notin(a, b)\left(\mathbb{Z}+\frac{1}{2}\right) .\end{cases}
$$

Proof. To begin, we fix $x \in \mathbb{Z}$ and sum the statement of Lemma 1 over all $y \in \mathbb{Z}_{a}$, rescaled by a factor of $\sqrt{a}$ :

$$
\begin{aligned}
\frac{1}{\sqrt{a}} \sum_{y \in \mathbb{Z}_{a}}\left(\mathrm{~F}_{a} \mathrm{c}_{a}^{b}\right)(x+b y) & =\frac{1}{\sqrt{a}} \sum_{y \in \mathbb{Z}_{a}} \overline{\mathrm{m}_{a}^{x}(y) \mathrm{c}_{a}^{b}(y)}\left(\mathrm{F}_{a} \mathrm{c}_{a}^{b}\right)(x) \\
& =\left(\mathrm{F}_{a} \mathrm{c}_{a}^{b}\right)(x) \frac{1}{\sqrt{a}} \overline{\sum_{y \in \mathbb{Z}_{a}} \mathrm{c}_{a}^{b}(y) \mathrm{w}_{a}^{x y}} \\
& =\left(\mathrm{F}_{a} \mathrm{c}_{a}^{b}\right)(x) \overline{\left(\mathrm{F}_{a} \mathrm{c}_{a}^{b}\right)(-x)}
\end{aligned}
$$

Next, we note that Proposition 3 gives that $c_{a}^{b}$ is an even function on $\mathbb{Z}_{a}$, namely, $c_{a}^{b}(-t)=c_{a}^{b}(t)$ for all $t \in \mathbb{Z}_{a}$. As with any locally compact abelian group, the Fourier transform of an even function is even, and so $\left(\mathrm{F}_{a} \mathrm{c}_{a}^{b}\right)(-x)=\left(\mathrm{F}_{a} \mathrm{c}_{a}^{b}\right)(x)$. Simplifying, we obtain

$$
\left|\left(\mathrm{F}_{a} \mathrm{c}_{a}^{b}\right)(x)\right|^{2}=\frac{1}{\sqrt{a}} \sum_{y \in \mathbb{Z}_{a}}\left(\mathrm{~F}_{a} \mathrm{c}_{a}^{b}\right)(x+b y) .
$$

To continue, we use the easily proven fact that

$$
\sum_{t \in \mathbb{Z}_{a}} f(b t)=\underset{\left\{x \in \mathbb{Z}_{a},(a, b) \mid x\right\}}{(a, b) \sum_{x} f(x)}
$$

for any $f \in \ell\left(\mathbb{Z}_{a}\right)$ and for any $b \in \mathbb{Z}$. Applying this fact when $f=\mathrm{F}_{a} \mathrm{M}_{a}^{-x} \mathrm{c}_{a}^{b}$ gives

$$
\begin{aligned}
\left|\left(\mathrm{F}_{a} \mathrm{c}_{a}^{b}\right)(x)\right|^{2} & =\frac{1}{\sqrt{a}} \sum_{y \in \mathbb{Z}_{a}}\left(\mathrm{~T}_{a}^{-x} \mathrm{~F}_{a} \mathrm{c}_{a}^{b}\right)(b y) \\
& =\frac{1}{\sqrt{a}} \sum_{y \in \mathbb{Z}_{a}}\left(\mathrm{~F}_{a} \mathrm{M}_{a}^{-x} \mathrm{c}_{a}^{b}\right)(b y) \\
& =\frac{(a, b)}{\sqrt{a}} \sum_{\left\{z \in \mathbb{Z}_{a},(a, b) \mid z\right\}}\left(\mathrm{F}_{a} \mathrm{M}_{a}^{-x} \mathrm{c}_{a}^{b}\right)(z) .
\end{aligned}
$$

Next, we apply the Poisson summation formula. Specifically, in terms of Proposition 2, taking " $a$," " $b$," and " $f$ " to be $a /(a, b),(a, b)$, and $\mathrm{F}_{a} \mathrm{M}_{a}^{-x} \mathrm{c}_{a}^{b}$, respectively, yields

$$
\begin{aligned}
\left|\left(\mathrm{F}_{a} \mathrm{c}_{a}^{b}\right)(x)\right|^{2} & =\frac{(a, b)}{\sqrt{a}} \frac{\sqrt{a /(a, b)}}{\sqrt{(a, b)}} \sum_{\left\{t \in \mathbb{Z}_{a},[a /(a, b)] \mid t\right\}}\left(\mathrm{M}_{a}^{-x} \mathrm{c}_{a}^{b}\right)(t) \\
& =\sum_{u=0}^{(a, b)-1}\left(\mathrm{M}_{a}^{-x} \mathrm{c}_{a}^{b}\right)\left(\frac{a u}{(a, b)}\right) \\
& =\sum_{u=0}^{(a, b)-1} \mathrm{w}_{a}^{-x a u /(a, b)}(-1)^{b a u /(a, b)} \mathrm{w}_{a}^{b a^{2} u^{2} / 2(a, b)^{2}}
\end{aligned}
$$

To simplify this expression, we note that

$$
\mathrm{w}_{a}^{-x a u /(a, b)}=\mathrm{e}^{-2 \pi \mathrm{i} x a u / a(a, b)}=\mathrm{e}^{-2 \pi \mathrm{i} x u /(a, b)} .
$$


Furthermore, since $(-1)^{u^{2}}=(-1)^{u}$ for all $u \in \mathbb{Z}$, then

$$
\mathrm{w}_{a}^{b a^{2} u^{2} / 2(a, b)^{2}}=\mathrm{e}^{\pi \mathrm{i} b a^{2} u^{2} / a(a, b)^{2}}=(-1)^{u a b /(a, b)^{2}} .
$$

Thus,

$$
\left|\left(\mathrm{F}_{a} \mathrm{c}_{a}^{b}\right)(x)\right|^{2}=\sum_{u=0}^{(a, b)-1} \mathrm{e}^{-2 \pi \mathrm{i} x u /(a, b)}(-1)^{u a b[(a, b)+1] /(a, b)^{2}} .
$$

To conclude, note that if $a$ and $b$ do not have a common power of two, then either $a /(a, b), b /(a, b)$ or $(a, b)+1$ is even. Our expression for $\left|\left(\mathrm{F}_{a} \mathrm{c}_{a}^{b}\right)(x)\right|^{2}$ is a geometric series:

$$
\begin{aligned}
\left|\left(\mathrm{F}_{a} c_{a}^{b}\right)(x)\right|^{2} & =\sum_{u=0}^{(a, b)-1} \mathrm{e}^{-2 \pi \mathrm{i} x u /(a, b)} \\
& = \begin{cases}(a, b), & \frac{x}{(a, b)} \in \mathbb{Z}, \\
0, & \frac{x}{(a, b)} \notin \mathbb{Z},\end{cases} \\
& = \begin{cases}(a, b), & x \in(a, b) \mathbb{Z}, \\
0, & x \notin(a, b) \mathbb{Z} .\end{cases}
\end{aligned}
$$

If, on the other hand, $a$ and $b$ have a common power of two, then $a /(a, b), b /(a, b)$, and $(a, b)+1$ are all odd. In this case, we obtain a slightly different geometric series:

$$
\begin{aligned}
\left|\left(\mathrm{F}_{a} \mathrm{c}_{a}^{b}\right)(x)\right|^{2} & =\sum_{u=0}^{(a, b)-1}(-1)^{u} \mathrm{e}^{-2 \pi \mathrm{i} x u /(a, b)} \\
& =\sum_{u=0}^{(a, b)-1} \mathrm{e}^{-2 \pi \mathrm{i}[x /(a, b)-1 / 2] u} \\
& = \begin{cases}(a, b), & \frac{x}{(a, b)}-1 / 2 \in \mathbb{Z}, \\
0, & \frac{x}{(a, b)}-1 / 2 \notin \mathbb{Z},\end{cases} \\
& = \begin{cases}(a, b), & x \in(a, b)\left(\mathbb{Z}+\frac{1}{2}\right), \\
0, & x \notin(a, b)\left(\mathbb{Z}+\frac{1}{2}\right) .\end{cases}
\end{aligned}
$$

We also have the following near-immediate corollary.

Corollary 1. For any $a \in \mathbb{Z}^{+}$and any $x_{1}, x_{2}, y_{1}, y_{2} \in \mathbb{Z}$, we have $\left|\left\langle\mathrm{m}_{a}^{x_{1}} \mathrm{c}_{a}^{y_{1}}, \mathrm{~m}_{a}^{x_{2}} \mathrm{c}_{a}^{y_{2}}\right\rangle\right|^{2}=a\left(a, y_{1}-y_{2}\right)$ when either

(i) a and $y_{1}-y_{2}$ do not have a common power of two and $x_{1}-x_{2}$ is an integer multiple of $\left(a, y_{1}-y_{2}\right)$, or

(ii) $a$ and $y_{1}-y_{2}$ have a common power of two and $x_{1}-x_{2}$ is a half-integer multiple of $\left(a, y_{1}-y_{2}\right)$,

and $\left\langle\mathrm{m}_{a}^{x_{1}} \mathrm{c}_{a}^{y_{1}}, \mathrm{~m}_{a}^{x_{2}} \mathrm{c}_{a}^{y_{2}}\right\rangle=0$, otherwise. In particular, when $a=$ $p$ is prime,

$$
\left|\left\langle\mathrm{m}_{p}^{x_{1}} \mathrm{c}_{p}^{y_{1}}, \mathrm{~m}_{p}^{x_{2}} \mathrm{c}_{p}^{y_{2}}\right\rangle\right|^{2}= \begin{cases}p^{2}, & \text { if } y_{1}=y_{2}, x_{1}=x_{2}, \\ p, & \text { if } y_{1} \neq y_{2}, \\ 0, & \text { if } y_{1}=y_{2}, x_{1} \neq x_{2},\end{cases}
$$

where all equivalences between either $x_{1}$ and $x_{2}$, or $y_{1}$ and $y_{2}$, are taken $\bmod p$.
Proof. For any $x_{1}, x_{2}, y_{1}, y_{2} \in \mathbb{Z}$,

$$
\begin{aligned}
\left|\left\langle\mathrm{m}_{a}^{x_{1}} \mathrm{c}_{a}^{y_{1}}, \mathrm{~m}_{a}^{x_{2}} \mathrm{c}_{a}^{y_{2}}\right\rangle\right|^{2} & =\left|\left\langle\mathrm{c}_{a}^{y_{1}-y_{2}}, \mathrm{~m}_{a}^{x_{2}-x_{1}}\right\rangle\right|^{2} \\
& =a\left|\left(\mathrm{~F}_{a} \mathrm{c}_{a}^{y_{1}-y_{2}}\right)\left(x_{2}-x_{1}\right)\right|^{2} .
\end{aligned}
$$

Returning to the issue brought up at the end of the previous section, the level of "uncorrelation" in our tight frames is

$$
\max _{\left(x_{1}, y_{1}\right) \neq\left(x_{2}, y_{2}\right)}\left|\left\langle\mathrm{m}_{a}^{x_{1}} \mathrm{c}_{a}^{y_{1}}, \mathrm{~m}_{a}^{x_{2}} \mathrm{c}_{a}^{y_{2}}\right\rangle\right|=\sqrt{a b}
$$

where $b$ is the largest proper divisor of $a$. As such, the "best-" modulated chirp frames are obtained when $a$ is prime, a fact which was originally noted in [1].

\section{FOURIER TRANSFORMS OF FINITE CHIRPS}

In our final results, we compute the actual values of the Fourier transforms of our finite chirps. Remarkably, we see that the Fourier transform of one chirp is a decimated version of another chirp.

Theorem 1. If $a \in \mathbb{Z}^{+}, b \in \mathbb{Z}$ do not have a common power of two, then

$$
\frac{\left(\mathrm{F}_{a} \mathrm{c}_{a}^{b}\right)(x)}{\left(\mathrm{F}_{a} \mathrm{c}_{a}^{b}\right)(0)}= \begin{cases}\mathrm{c}_{a}^{-b d^{2}}\left(\frac{x}{(a, b)}\right), & x \in(a, b) \mathbb{Z}, \\ 0, & x \notin(a, b) \mathbb{Z},\end{cases}
$$

where $d \in \mathbb{Z}, b d=(a, b) \bmod a$.

Proof. Take any $x \in \mathbb{Z}$. If $(a, b)$ does not divide $x$, then Proposition 5 implies $\left(\mathrm{F}_{a} \mathrm{c}_{a}^{b}\right)(x)=0$. If $(a, b)$ does divide $x$, take $t \in \mathbb{Z}$ such that $x=(a, b) t$, and take $c, d \in \mathbb{Z}$ such that $a c+b d=(a, b)$. Since $\mathrm{F}_{a} \mathrm{c}_{a}^{b}$ is well defined over $\mathbb{Z}_{a}$,

$$
\begin{aligned}
\left(\mathrm{F}_{a} \mathrm{c}_{a}^{b}\right)(x) & =\left(\mathrm{F}_{a} \mathrm{c}_{a}^{b}\right)((a, b) t) \\
& =\left(\mathrm{F}_{a} \mathrm{c}_{a}^{b}\right)(a c t+b d t) \\
& =\left(\mathrm{F}_{a} c_{a}^{b}\right)(b d t) .
\end{aligned}
$$

Applying Lemma 1, with " $x$ " and " $y$ " taken to be 0 and $d t$, respectively, we have

$$
\left(\mathrm{F}_{a} \mathrm{c}_{a}^{b}\right)(x)=\overline{\mathrm{m}_{a}^{0}(d t) \mathrm{c}_{a}^{b}(d t)}\left(\mathrm{F}_{a} \mathrm{c}_{a}^{b}\right)(0) .
$$

Next, note that Proposition 5 implies $\left(\mathrm{F}_{a} \mathrm{c}_{a}^{b}\right)(0) \neq 0$. Also, $\mathrm{m}_{a}^{0}(d t)=1$. Thus, by Proposition 3,

$$
\frac{\left(\mathrm{F}_{a} \mathrm{c}_{a}^{b}\right)(x)}{\left(\mathrm{F}_{a} \mathrm{c}_{a}^{b}\right)(0)}=\overline{\mathrm{c}_{a}^{b}(d t)}=\mathrm{c}_{a}^{-b d^{2}}(t)=\mathrm{c}_{a}^{-b d^{2}}\left(\frac{x}{(a, b)}\right) .
$$

Theorem 2. Let $a \in \mathbb{Z}^{+}, b \in \mathbb{Z}$ have a common power of two, that is, let $a=2^{m} \alpha$ and $b=2^{m} \beta$ with $\alpha$ and $\beta$ odd. Then,

$$
\begin{aligned}
& \frac{1}{2^{m / 2}} \frac{\left(\mathrm{F}_{a} \mathrm{c}_{a}^{b}\right)(x)}{\left(\mathrm{F}_{\alpha} \mathrm{c}_{\alpha}^{\beta}\right)(0)} \\
& \quad= \begin{cases}\mathrm{c}_{\alpha}^{-\beta \delta^{2}}\left(\frac{(x-a / 2)}{(a, b)}\right), & x \in(a, b)\left(\mathbb{Z}+\frac{1}{2}\right), \\
0, & x \notin(a, b)\left(\mathbb{Z}+\frac{1}{2}\right),\end{cases}
\end{aligned}
$$

where $\delta \in \mathbb{Z}, \beta \delta=(\alpha, \beta) \bmod \alpha$. 
Proof. Take any $x \in \mathbb{Z}$. If $x$ is not a half-integer multiple of $(a, b)$, then Proposition 5 implies $\left(\mathrm{F}_{a} \mathrm{c}_{a}^{b}\right)(x)=0$. If $x$ is a halfinteger multiple of $(a, b)$, let $t=(x-a / 2) /(a, b)$, so that $x=$ $(a, b) t+a / 2$. Note that since $a$ and $b$ have a common factor of two, then $a / 2(a, b) \in \mathbb{Z}+1 / 2$. Since $x /(a, b) \in \mathbb{Z}+1 / 2$ as well, then $t \in \mathbb{Z}$.

Take $c, d \in \mathbb{Z}$ such that $a c+b d=(a, b)$. Since $a=2^{m} \alpha$ and $b=2^{m} \beta$ with $\alpha$ and $\beta$ odd, we have

$$
2^{m}(\alpha c+\beta d)=(a, b)=\left(2^{m} \alpha, 2^{m} \beta\right)=2^{m}(\alpha, \beta),
$$

and thus $\alpha c+\beta d=(\alpha, \beta)$. Letting $\delta=d$, we therefore have $\beta \delta=(\alpha, \beta) \bmod \alpha$.

Continuing, note that since $\mathrm{F}_{a} \mathrm{c}_{a}^{b}$ is well defined over $\mathbb{Z}_{a}$,

$$
\begin{aligned}
\left(\mathrm{F}_{a} \mathrm{c}_{a}^{b}\right)(x) & =\left(\mathrm{F}_{a} \mathrm{c}_{a}^{b}\right)\left((a, b) t+\frac{a}{2}\right) \\
& =\left(\mathrm{F}_{a} \mathrm{c}_{a}^{b}\right)\left(a c t+b \delta t+\frac{a}{2}\right) \\
& =\left(\mathrm{F}_{a} \mathrm{c}_{a}^{b}\right)\left(b \delta t+\frac{a}{2}\right) .
\end{aligned}
$$

Applying Lemma 1, with " $x$ " and " $y$ " taken to be $a / 2$ and $\delta t$, respectively, we have

$$
\left(\mathrm{F}_{a} \mathrm{c}_{a}^{b}\right)(x)=\overline{\mathrm{m}_{a}^{a / 2}(\delta t) \mathrm{c}_{a}^{b}(\delta t)}\left(\mathrm{F}_{a} \mathrm{c}_{a}^{b}\right)\left(\frac{a}{2}\right)
$$

To continue, note that for any $u \in \mathbb{Z}$,

$$
\mathrm{m}_{a}^{a / 2}(u)=\mathrm{w}_{a}^{(a / 2) u}=\mathrm{e}^{2 \pi \mathrm{i}(a / 2) u / a}=(-1)^{u}=(-1)^{\beta u}
$$

since $\beta$ is odd. Similarly, since $b$ is even,

$$
\begin{aligned}
\mathrm{c}_{a}^{b}(u) & =(-1)^{b u} \mathrm{w}_{a}^{b u^{2} / 2} \\
& =\mathrm{e}^{\pi \mathrm{i} b u^{2} / a} \\
& =\mathrm{e}^{\pi \mathrm{i} 2^{m} \beta u^{2} /\left(2^{m} \alpha\right)} \\
& =\mathrm{e}^{\pi \mathrm{i} \beta u^{2} / \alpha}
\end{aligned}
$$

Taken together, we have that

$$
\mathrm{m}_{a}^{a / 2}(u) \mathrm{c}_{a}^{b}(u)=(-1)^{\beta u} \mathrm{e}^{\pi \mathrm{i} \beta u^{2} / \alpha}=\mathrm{c}_{\alpha}^{\beta}(u),
$$

and consequently,

$$
\left(\mathrm{F}_{a} \mathrm{c}_{a}^{b}\right)(x)=\overline{\mathrm{c}_{\alpha}^{\beta}(\delta t)}\left(\mathrm{F}_{a} \mathrm{c}_{a}^{b}\right)\left(\frac{a}{2}\right) .
$$

Similarly,

$$
\begin{aligned}
\left(\mathrm{F}_{a} \mathrm{c}_{a}^{b}\right)\left(\frac{a}{2}\right) & =\frac{1}{\sqrt{a}} \sum_{u \in \mathbb{Z}_{a}} \mathrm{c}_{a}^{b}(u) \mathrm{m}_{a}^{-a / 2}(u) \\
& =\frac{1}{\sqrt{a}} \sum_{u \in \mathbb{Z}_{a}} c_{\alpha}^{\beta}(u) \\
& =\frac{2^{m}}{\sqrt{2^{m} \alpha}} \sum_{u \in \mathbb{Z}_{\alpha}} c_{\alpha}^{\beta}(u) \\
& =2^{m / 2} \mathrm{~F}_{\alpha} \mathrm{c}_{\alpha}^{\beta}(0)
\end{aligned}
$$

and therefore,

$$
\left(\mathrm{F}_{a} \mathrm{c}_{a}^{b}\right)(x)=\overline{\mathrm{c}_{\alpha}^{\beta}(\delta t)} 2^{m / 2} \mathrm{~F}_{\alpha} \mathrm{c}_{\alpha}^{\beta}(0)
$$

Finally, as Proposition 5 implies that $\mathrm{F}_{\alpha} \mathrm{C}_{\alpha}^{\beta}(0) \neq 0$, then

$$
\frac{1}{2^{m / 2}} \frac{\left(\mathrm{F}_{a} \mathrm{c}_{a}^{b}\right)(x)}{\mathrm{F}_{\alpha} \mathrm{c}_{\alpha}^{\beta}(0)}=\overline{\mathrm{c}_{\alpha}^{\beta}(\delta t)}=\mathrm{c}_{\alpha}^{-\beta \delta^{2}}\left(\frac{(x-a / 2)}{(a, b)}\right),
$$

by Proposition 3.

As a final remark, we note that in each of these results, we have only determined the Fourier transforms of our chirps up to a scalar multiple. To resolve the matter further, one must explicitly determine the values $\mathrm{c}_{a}^{b}(0)$ for all $a$ and $b$ that do not share a common power of two. In fact, $c_{a}^{b}$ may be shown to be always $\sqrt{(a, b)}$ times an eighth root of unity. This problem was classically solved using sophisticated techniques of number theory involving special functions and complex analysis. However, the techniques presented in this paper may also be further developed to provide independent, elementary proofs of these same results [8].

\section{ACKNOWLEDGMENTS}

This work was partially supported by the National Science Foundation (DMS 0405376). The views expressed in this paper are those of the authors and do not reflect the official policy or position of the United States Air Force, Department of Defense, or the US Government.

\section{REFERENCES}

[1] X.-G. Xia, "Discrete chirp-Fourier transform and its application to chirp rate estimation," IEEE Transactions on Signal Processing, vol. 48, no. 11, pp. 3122-3133, 2000.

[2] S. Mann and S. Haykin, "The chirplet transform: A generalization of Gabor's logon transform," in Proceedings of Vision Interface, pp. 205-212, Calgary, Alberta, Canada, June 1991.

[3] B. C. Berndt and R. J. Evans, "The determination of Gauss sums," Bulletin of the American Mathematical Society (New Series), vol. 5, no. 2, pp. 107-129, 1981.

[4] L. Auslander and R. Tolimieri, "Is computing with the finite Fourier transform pure or applied mathematics?" Bulletin of the American Mathematical Society (New Series), vol. 1, no. 6, pp. 847-897, 1979.

[5] J. H. McClellan and T. W. Parks, "Eigenvalue and eigenvector decomposition of the discrete Fourier transform," IEEE Transactions on Audio and Electroacoustics, vol. 20, no. 1, pp. 66-74, 1972.

[6] N. Kaiblinger, Metaplectic representation, eigenfunctions of phase space shifts, and Gelfand-Shilov spaces for lca groups, Ph.D. thesis, University of Vienna, Vienna, Austria, 1999.

[7] T. Strohmer and R. W. Heath Jr., "Grassmannian frames with applications to coding and communications," Applied and Computational Harmonic Analysis, vol. 14, no. 3, pp. 257-275, 2003.

[8] M. Fickus, "An elementary proof of a generalized Schaar identity," preprint, 2005. 
Peter G. Casazza received his Ph.D. degree from the University of Iowa in 1972. He has been at the University of Missouri since 1983. He was a National Academy of Sciences Fellow to the Soviet Union from 1977 to 1978. He has been a Visiting Scholar at the Hebrew University of Jerusalem (1 year), Cambridge University, England (2 years), and Odense University,

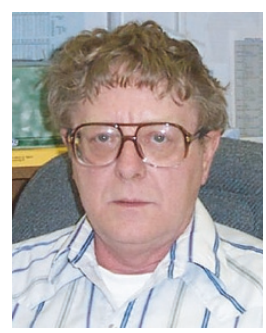
Denmark (1 year). He has won the Outstanding Greek Faculty Award, the Student Athlete Most Inspiring Professor Award, and the Kemper Excellence in Teaching Award from the University of Missouri. He has been the Vice President of the Missouri MAA and is an Associate Editor of the Journal of Math Analysis and Applications. His research has been supported by the National Science Foundation for the last 26 years. His research interests are in applications of harmonic analysis, functional analysis, operator theory, number theory, and so forth to problems in Hilbert space frame theory-especially industrial applications.

Matthew Fickus received his Ph.D. degree in mathematics from the University of Maryland, College Park in 2001. He then spent three years at Cornell University in a postdoctoral position, supported by a VIGRE grant from the National Science Foundation. In 2004, he began his current position as an Assistant Professor at the Air Force Institute of Technology. His current research interests include Fourier se-

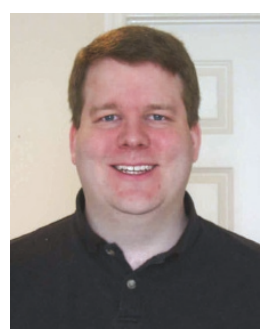
ries, wavelets, and frames, with an emphasis upon signal processing applications. 